\title{
Histoires des techniques et des mobilités
}

\section{Konstantinos Chatzis}

\section{(2) OpenEdition \\ 1 Journals}

\section{Édition électronique}

URL : http://journals.openedition.org/artefact/725

DOI : $10.4000 /$ artefact.725

ISSN : 2606-9245

Éditeur :

Association Artefact. Techniques histoire et sciences humaines, Presses universitaires du Midi

\section{Édition imprimée}

Date de publication : 15 juin 2017

Pagination : 213-214

ISBN : 978-2-7535-6525-8

ISSN : 2273-0753

\section{Référence électronique}

Konstantinos Chatzis, "Histoires des techniques et des mobilités ", Artefact [En ligne], 5 | 2016, mis en ligne le 15 novembre 2017, consulté le 24 septembre 2020. URL : http://journals.openedition.org/ artefact/725 ; DOI : https://doi.org/10.4000/artefact.725

\section{(c) (i) $\odot$}

Artefact, Techniques, histoire et sciences humaines est mise à disposition selon les termes de la Licence Creative Commons Attribution - Pas d'Utilisation Commerciale - Pas de Modification 4.0 International. 
Depuis plusieurs années déjà, sous les effets conjugués d'un nombre de facteurs aussi variés que le problème de la congestion, celui de la pollution de l'air, la question des dépenses énergétiques et, plus récemment, celle du réchauffement climatique, le secteur des transports a entrepris des mutations d'une grande ampleur à l'échelle du globe. Ces changements, qui ont affecté pratiquement tous les éléments du secteur, touché ainsi dans ses aspects techniques mais aussi organisationnels et professionnels, économiques et sociaux, politiques et juridiques, se sont cristallisés autour d'une série d'enjeux transversaux, comme la promotion de modes de transports alternatifs à la voiture privée ou la mise en avant d'une vision multimodale (et globale) de la chaîne des déplacements.

Il n'y a pas que le secteur qui connaisse ces vagues de changements. Sans qu'on puisse établir de connexion directe entre les deux ordres de phénomènes, force est de constater que depuis une vingtaine d'années des évolutions importantes sont enregistrées également dans le regard que les sciences sociales en général, la discipline historique en particulier, portent sur le phénomène du transport. Grâce aux efforts de plus en plus coordonnés de nombreux membres de l'univers académique à travers le monde, organisés désormais en associations et groupes de travail, formant des réseaux internationaux et bénéficiant même de supports dédiés de publication ${ }^{1}$, on assiste à l'émergence de nouvelles façons d'envisager l'objet « transport ». $\mathrm{D}^{\prime}$ après les termes de deux historiens français qui contribuent à ce renouveau intellectuel, c'est la notion de mobilité qui fonctionne comme «1'"opérateur", intellectuel et institutionnel, qui déplace aujourd'hui les problématiques de l'histoire des transports dans le monde académique ${ }^{2} »$. Sur les acquis d'une histoire des transports « à l'ancienne » dont les différents chapitres reprenaient plus au moins les différents modes spécifiques - la route, le chemin de fer, le bateau et l'avion - a émergé une nouvelle histoire moins soucieuse du découpage des modes et des infrastructures et plus sensible aux thématiques transversales, comme la sécurité et la mobilité durable, pour reprendre les thèmes mis en avant par l'Association internationale d'histoire de la mobilité $\mathrm{T}^{2} \mathrm{M}$ lors de sa quatrième conférence annuelle en 2006 sur les sites de Paris et de Marne-la-Vallée. Notons ici que si les changements dont le secteur des transports lui-même est depuis plusieurs années le théâtre ont pu participer à cet élargissement de la focale, des mutations internes à la discipline historique ont joué également leur part. Il suffit de penser à l'intérêt que la discipline accorde désormais aux questions des représentations (politiques

1. Citons ainsi la création, en novembre 2003, de $\mathrm{T}^{2} \mathrm{M}$ (International Association for the History of Transport, Traffic $\mathcal{E}$ Mobility), « an association of scholars, practitioners and concerned citizens who seek to encourage and promote historically informed understanding of transport, traffic and mobility " [https :/ /t2m.org/], qui publie un recueil annuel (Yearbook) intitulé Mobility in History (sept volumes parus jusqu'en 2016, [http://t2m.org/publications/yearbook/]). Citons aussi deux revues récentes : Mobilities ( $1^{\mathrm{er}}$ numéro en 2006, [http://www.tandfonline.com/toc/rmob20/current]) et Transfers : The Interdisciplinary Journal of Mobility Studies (fondée en 2011, [http:/ /journals.berghahnbooks.com/ transfers]).

2. Mathieu Flonneau et Vincent Guigueno (dir.), De l'histoire des transports à l'histoire de la mobilité?, Rennes, PUR, 2009, p. 12. 
et autres), de l'imaginaire ou à celle des sensibilités, et qui traversent à des degrés divers plusieurs des comptes rendus regroupés ici.

Le lecteur est invité à se faire une idée des nouvelles relations que les historiens cultivent avec les transports ces dernières années à travers les comptes rendus de cinq ouvrages qui parlent tour à tour $1^{\circ} \mathrm{du}$ retour de la bicyclette dans les villes européennes et de sa place dans l'évolution de la mobilité urbaine sur la longue durée, $2^{\circ}$ des autoroutes urbaines (américaines), leurs acteurs et leurs visions (souvent) contradictoires sur ce qu'est une "ville bonne " et sur le rôle que l'urban freeway est destinée à jouer dans sa fabrication, $3^{\circ}$ des rapports noués entre les mobilités d'une part, la liberté, la démocratie et le politique de l'autre, $4^{\circ}$ de l'influence de l'aéronautique sur les arts et la culture, et $5^{\circ}$ de l'imaginaire de l'aviation pionnière. Les comptes rendus qui suivent ne répondent pas à une commande et n'obéissent pas à un plan préconçu. Ils forment un ensemble plutôt hétéroclite tant du point de vue du contenu des livres dont ils rendent compte et des sensibilités des auteurs qui les signent (et dont certains ne se voient pas nécessairement comme membres de la «famille » issue du mobility turn). Mais pris ensemble, et grâce même à l'hétérogénéité qui les caractérise, ils donnent à voir collectivement un champ intellectuel dynamique et en pleine évolution. 